\title{
Article@Virology
}

\section{An Analysis of Quality Management in Vaccine Manufacturers of China Based on the New Vaccine Administration Law}

Xue Min Ji, Xiang Qun Li, Cui Liu*

Beijing Minhai Biological Technology Co., Ltd., Beijing, 102629, P.R.China

\begin{abstract}
With the application of new technologies in development and production, the quality of vaccines in China had been constantly improved, and vaccines manufactured in China were being supplied to international markets while meeting the domestic immunization program need. Meanwhile, the quality management performance of vaccine manufacturers in China was continually improving. The implementation of the Vaccine Administration Law of the People's Republic of China had imposed legal systematic requirements on full lifecycle management of vaccines in development, production and supervision, which was of important milestone significance for guiding the development of China' s vaccine industry. Based on the understanding of this law, this article had discussed quality management of vaccines from four aspects including biosafety, application of new technologies for vaccine development and production, process changes and deviation management with practical work taken into account, intended to arouse readers'attention and discussions on this law and relevant issues and provide reference to vaccine manufacturers in production, development and product quality improvement.
\end{abstract}

Copyright@2012-2025 Published by Hong Kong Institute of Biologicals Standardization Limited. All rights reserved.

Article history: Submitted: 06/04/2020; Revised: 27/04/2020; Accepted: 30/04/2020

DOI: $10.21092 /$ jav.v9i2.88

Key Words: Vaccine Administration Law of the People's Republic of China, Vaccine, Biosafety, New technology, Process change, Deviation management

\footnotetext{
* Corresponding author, Senior Engineer, Major in Biopharmaceutical

E-mail: liucui@biominhai.com
} 


\section{Introduction}

Vaccines were a class of biological products prepared using biotechnologies with pathogenic microorganisms or their components and metabolites as starting materials and used for the prevention and treatment of corresponding human diseases. Vaccines administered to the human body might stimulate the immune system to generate specific humoral and (or) cellular immune responsed so as to get immunity to corresponding pathogenic microorganisms ${ }^{[1]}$.

Since 1919, China's vaccine development had a history of 100 years ${ }^{[2]}$. To date, there were 46 vaccine manufacturers in China, which could produce more than 60 vaccine products for 34 diseases in different populations, with an annual production capacity of more than 1 billion doses ${ }^{[3]}$.

After a century of development, China's vaccine industry, especially since the 40 years of reform and opening up, had made substantial progress in terms of production varieties, manufacturing scale, production technology, regulatory system, etc., the quality level had been continually improved, and more product varieties had been developed. China passed the assessment of the national regulatory system for vaccines conducted by the World Health Organization (WHO) in 2011 and 2014, and four product varieties, including hepatitis A vaccine, encephalitis B vaccine, influenza vaccine and polio vaccine, had passed the WHO pre-certification, indicating China-made vaccines had taken a role on the international stage ${ }^{[4]}$.

The Vaccine Administration Law of the People's Republic of China (hereinafter referred to as "Vaccine Administration Law") was the first comprehensive law on vaccine administration in the world, which, entered into force on December 1, 2019, was of important milestone significance for guiding the development of China's vaccine industry. This article was mainly discussed four aspects including biosafety, application of new technologies for vaccine development and production, process changes and deviation management.

\section{Introduction of the Vaccine Adminis- -tration Law}

Vaccines were mainly for use by healthy people, especially infants and toddlers. The quality of vaccines correlated closely to people's health and also to public health and safety and national security. In response to concerned of the masses and with a view to Fully implementing the requirements of General Secretary Xi Jinping on the "four strictest requirements" for food and medicine, fulfilling measures of the Party Central Committee and the State Council for strengthening the vaccine administration reform, integrating separate laws and regulations, enhancing the pertinence, timeliness and feasibility of legislation for vaccines, the Vaccine Administration Law had been formulated under the leadership of the National Medical Products 
Administration ${ }^{[5]}$.

The Vaccine Administration Law comprised 11 chapters and 100 articles. The Drug Administration Law of the People's Republic of China must be taken into account in understanding this law. The two laws had jointly stipulated provisions on the drug marketing authorization holder system, the drug traceability system and the pharmacovigilance system. Compared to the Drug Administration Law of the People's Republic of China, the Vaccine Administration Law mainly focused on specific requirements on vaccine administration according to characteristics of vaccine development, production and circulation and vaccination.

The institution and implementation of the Vaccine Administration Law had fully embodied the philosophy of "enhancing vaccine administration, guaranteeing vaccine quality and supply, standardizing prophylactic immunization, promoting the development of the vaccine industry and ensuring public health and safety". The implementation of the Vaccine Administration Law had brought about both opportunities and challenges to manufacturers. For example, Article 4 required "The State shall institute development planning and industrial policies for the vaccine industry to support development and structural optimization of the vaccine industry, encourage scale and intensive vaccine production, and constantly improve the production process and quality level of vaccines." Legalization of the on-site inspector mechanism would help strictly control vaccine production behaviors from the source (refer to Article 71 for detail). Article 68 required "The State shall practice a compulsory vaccine liability insurance system" and specified the establishment and implementation of vaccine insurance related regulations, the compensation system for vaccines would be further clarified. The law had also specified the responsibilities and roles of industry associations. It was foreseeable that industry associations would play a more active role in promoting self-discipline of the vaccine industry and guiding manufacturers in production and development according to law (refer to Article 13).

\section{An analysis of quality management in vaccine manufacturers based on the Vaccine Administration Law}

\subsection{Biosafety}

Biosafety was an important part of national security, which refered to safety problems with an impact on ecological environment and human health resulting from the whole process of biotechnology from research, development, production to practical application ${ }^{[6]}$. Article 11 of the Vaccine Administration Law specifies that "In the process of vaccine development, production and testing, a sound biosafety management system shall be established to 
strictly control biosafety risks".

Regarding biosafety, both domestic and foreign regulatory authorities had released relevant guidelines and technical requirements. Chinese Pharmacopoeia (Volume III) (2015 edition) ${ }^{[1]}$ and Good Manufacturing Practices (GMP) ${ }^{[7]}$ had prescribed requirements on matters involving biosafety including biological materials, production management, etc. The Determining the Need for and Content of Environmental Assessments for Gene Therapies, Vectored Vaccines, and Related Recombinant Viral or Microbial Products issued by the U.S. Food and Drug Administration (FDA) ${ }^{[8]}$ had put forward relevant technical requirements for environmental assessments for biologically active products.

Chinese Pharmacopoeia (Volume III) (2015 edition) had included 16 attenuated live vaccines including live attenuated polio vaccine and live attenuated encephalitis $B$ vaccine. In the extensive used of live attenuated vaccines, related biosafety issues including whether attenuated viral strains would result in the spread of vaccine viruses in use or during excretion from the human body to the nature, and whether the virulence of attenuated strains would be restored during transmission were still certain researchers' major concerns. Studied had shown that a tiny minority of immunodeficient or immunocompromised children might develop vaccine associated poliomyelitis (VAPP) following oral administration of live attenuated polio vaccine (OPV) or exposure to OPV vaccinated individuals, and presented the same clinical characteristics as poliomyelitis [9-10]. Xinyu Liu et al. characterized the spatial structure of $\mathrm{E}$ protein of live encephalitis B vaccine attenuated strain SA14-14-2 and virulent strain SA14 using $\mathrm{X}$-ray crystallography to investigate the mechanism of virus attenuation and simulated the transmission process of encephalitis B virus in nature by passing SA14-14-2 virus stain in mosquitoes, pigs and mosquito hosts, analyzing the viral genome sequence and virulence. The results showed that the spatial aggregation structure of $E$ protein of encephalitis B is associated with its individual virulence ${ }^{[11-12]}$. The above studies could be used for reference in studies on the mechanism of attenuation of attenuated live vaccine virus strains and their impact on human beings and the environment in use.

Biosafety not only related to well-known substances of biological origin, sterilization /inactivation processes, sterility, cross contamination for development and production, and also to the potential impact of active ingredients of biological products secreted by recipients to the nature on the ecological environment and human health, e.g., used of attenuated live vaccines, vectored vaccines, genetically engineered live attenuated vaccines, etc. Safety, 
effectiveness, quality controllability and environmental friendliness had become important concerns in the development and production process of live attenuated vaccines. Continual attention and investigation of the persistent impact of viral and bacterial strains used in biological products on human beings and the environment was required throughout the life cycle of vaccines from development to marketing to earnestly ensured the safety and effectiveness of vaccine quality manage-ment.

Biosafety risks involved in production elements including biological materials, virus inactivation process and cross contamination as mentioned in Chinese Pharmacopoeia and GMP could be effectively controlled by implementing GMP in vaccine manufacturers in China, and training and regulations on correctly cultivating the awareness of safety protection in production and taking appropriate safety protection measures had been continually improved and standardized [13-14]. However, in China, studied on the impact of biological products for human used on the ecological environment had just started, and related fundamental studied, particularly studied on the causes, nature, assessment and prevention of biosafety risks, need to be strengthened ${ }^{[6]}$.

\subsection{Used of new technologies in the development and production of vaccines}

Vaccines could prevent infectious diseases by stimulating the human immune system. With the development of new technologies such as new antigen synthesis processes, new adjuvants and vaccine vectors and human immune mechanism research and the application of new processes and new materials in production, the development of new vaccines and new manufacturing processes and the production and use of new materials had become the hotspot of development. New vaccines included new vaccines for diseases without effective prophylactic means, new adjuvanted vaccines, vaccines manufactured using new expression systems, combination vaccine, etc. [15-19]. Article 14 of the Vaccine Administration Law specified that "Support shall be provided for the development of new vaccines including combination vaccines".

In the U.S. FDA Guidance for the Evaluation of Combination Vaccines, a combination vaccine was defined as a combination of two or more live organisms, inactivated organisms or purified antigens combined either by the manufacturer or mixed immediately before administration and intended to prevent multiple diseases or prevent one disease caused by different strains or serotypes of the same organism. Vectored vaccines and conjugated vaccines 
were combination baccines ${ }^{[20]}$.

In the development of new vaccines, it wasn't advisable to make copies of new vaccines had been launched onto the market in foreign countries; rather, the prevalence of diseases and human immunity in China should been taken into account to use new technologies to develop new vaccines for infectious diseases with extensive prevalence, high incidence and great harm, and, on the basis of full studies, used effective quality management systems to enhance the scientificity, practicability and feasibility of vaccine development, so as to meet the actual needs of immunization in China. For post-approval vaccine products, it had become a trend to use new technologies and new processes to optimize their processes so as to constantly improve product quality and output. The manufacturing process maturity and quality control level of vaccine products directly determined product quality. Successful development and marketing of a product didn't signify the end of process development. With the accumulation of manufacturing batch data and the accumulation and analysis of post-marketing safety and effectiveness evaluation data, the manufacturing processes should be optimized and improved on the basis of continuous in-depth understanding of the product process and quality.

Hailun Ma from the U.S. FDA discovered a new RNA rhabdovirus from the $\mathrm{Sf} 9$ cell line (derived from Spodoptera frugiperda), verified the presence of the virus in the Sf9 by the observation of the supernatant samples of Sf9 cells with transmission electron microscope, and identified transcribed rhabdovirus like sequences in Sf9 cells ${ }^{[21-22]}$. The above studies indicated attention should be paid to the risks of new exogenous agents when using new protein expression technologies, e.g., Baculovirus Expression Vector System (BEVS), to ensure product quality safety. In using new vaccine manufacturing technologies, many aspects including risks of potential exogenous agents should be cautiously assessed.

\subsection{Process changed}

Article 15 of the Vaccine Administration Law specified that "The State shall encourage vaccine marketing authorization holders to increase capital investment to development and innovation, optimize production processes, elevate quality control levels and propel the progress of vaccine technologies." Vaccine manufacturers' innovation in production management didn't include solely use of new technologies. In-depth learning of quality management systems and application of new quality management approaches were also important.

Technical guidelines issued by the Center for Drug Evaluation (CDE) of National Medical Products Administration including the Technical Guideline on the Investigation of Post-approval CMC Changes to Biological Products (Draft for Comment) [23] 
and Technical Guideline on the Investigation of Post-approval Manufacturing Process Changes to Vaccines (Draft for Comment) ${ }^{[24]}$ had all referenced state-of-the-art management philosophy of developed countries to keep in line with international practices for change management, and introduced quality system parts (Q1-Q12) of the International Conference on Harmonization ( $\mathrm{ICH})$, among which, relevant cutting-edge management tools in ICH Q12 include Post-Approval Change Management Protocols (PACMPs), Established Conditions (ECs), Design Space, etc. The learning and used of cutting-edge change management tools would help standardize the research on manufacturers' process changes and accelerate the approval of changes.

Changes were an important means to keep product manufacturing processes advanced and improve product quality and manufacturing efficiency. With the implementation of the Vaccine Administration Law and the harmonization with ICH regulatory requirements, vaccine manufacturers should actively learnt relevant regulations and technical guidelines. Data should be continually accumulated throughout the lifecycles of products to deepen the understanding of products and processes and comprehensive considerations should be made based on risk assessment principles, so as to establish quality control systems meeting product needs, improved the stability of product processes and continually upgrade manufacturing processes for improving the safety, effectiveness based on the manufacturing efficiency of vaccines [25-26].

\subsection{Deviation management}

Deviation management was an important element of full lifecycle management of vaccines. The Vaccine Administration Law, on the basis of emphasizing the concept of full life cycle management, clarified, in Article 25 and Article 31, relevant requirements including continually strengthening deviation management and truthfully documenting manufacturing process deviation management measures. It was foreseeable that, in the future, management of major deviations would be linked with batch release of vaccines.

Under the influence of multiple factors including process changed, manufacturing environment changed and equipment depreciation, deviations were inevitable during pharmaceutical productions; different types of deviations would exert different impacts on product quality; for deviations with substantial or potential influenced on product quality, the causes should be identified and corrective actions should be taken. Manufacturers must control deviations in production. Correct recognition, identification and management of deviations were the prerequisite for ensuring the normal operation of the quality management for pharmaceutical production. The current 
GMP incorporated deviation management into quality control and quality assurance, required that manufacturers should establish operating procedures for deviation handling and specifies deviation reporting, documentation, investigation, handling and corrective actions as well as making of corresponding records ${ }^{[7]}$.

"The quality of products depends on production rather than inspection" was the core principle of the quality assurance system, which embodied the necessity of manufacturing in-process controls ${ }^{[27]}$. Both ICH Q8 and the U.S. FDA Investigatiing Out-of-Specification(OOS) Test Results for Pharmaceutical Production ${ }^{[28]}$ had proposed technical requirements on deviation management. Taking the Vaccine Administration Law in to account and according to requirements in relevant domestic and foreign technical guidelines, we had drafted the Procedure for Deviation Handling and Management, which had specified the responsibilities of each department in deviation handling. In this document, deviations, defined as deviations from approved procedures, instructions or established standards, refer to unscheduled events that do not comply with established SOPs, master batch records, laws and regulations, validation systems and testing methods/specifications or other criteria. The events may have an impact on the purity, strength, quality, efficacy or safety of manufacturing materials and may also affect regulatory compliant and validated equipment or processes used for production, storage or product distribution.

Depending on the influence on product quality, deviations were classified into secondary deviations, primary deviations and major deviations. Procedures for the handling of a deviation in production included: deviation occurrence, identifi-cation, reporting, determining whether emergency actions were needed, reporting the deviation to QA, deviation classification, investigation of causes, impact assessment, proposing corrective actions and preventive actions, approving corrective actions and preventive actions, completing correction, completing the deviation report, documentation and archiving of the deviation, deviation closure, and deviation reviewed analysis. To avoid potential quality risks and ensured the effectiveness of vaccine production quality management system, scientific deviation identification and management procedures should be established, training on deviation management should be strengthened, and comprehensive corrective actions and preventive actions should be taken.

\section{Epilogue}

The Vaccine Administration Law was the first comprehensive law on vaccine administration in the world. Its promulgation was of important milestone significance for guiding the development of China's vaccine industry. Vaccine manufacturers and 
developers should actively learn the Vaccine Administration Law, adapted to relevant requirements in this law and earnestly strengthen the building of vaccine manufacturing quality capacities to ensure vaccine products are safe, effective and quality-controllable.

Based on the authors' understanding of the Vaccine Administration Law, this article had, with this law, considered the latest domestic and foreign study results as well as relevant technical guidelines, provided an analysis of vaccine quality management from four aspects including biosafety, used of new technologies in vaccine development and production, process changes and deviation management, intended to aroused readers' attention and discussions on this law and relevant issues and provided reference to vaccine manufacturers in production, development and product quality improvement.

\section{Reference}

[1]. Chinese Pharmacopoeia Commission. Chinese Pharmacopoeia (Volume III) (2015 edition) [S]. 2015-06.

[2]. Qiang Z. RESEARCH ON EXPORT OF CHINESE BIOLOGICAL PRODUCTS [D]. University of International Business and Economics, 2016.

[3]. Jing LS. Vaccine Quality Management and Regulatory Inspection $[\mathrm{J}]$. Chinese Journal of Drug Evaluation, 2014,31(01):48-50+60.

[4]. Min L, Huan Y. Introduction of WHO's
Vaccine Prequalification Programme and Discussion on the Scientific Review Issues Identified During Vaccines Assessment by WHO [J]. Pharmaceutical Biotechnology, 2015,22(03):189-192.

[5]. State Administration for Market Regulation. The General Administration of Market Supervision announcement on public comment on Vaccine Administration Law of the People's Republic of China (Draft for Comment).[EB/OL].(2018-11-11)

[2020-04-05].

http://www.samr.gov.cn/hd/zjdc/201811/t2018 $1111291910 . \mathrm{htm}$

[6]. Qing HC, Min L, Jian HL, et al. General consideration for the environmental risk assessment for genetically modified live attenuated vaccines $[\mathrm{J}]$. Chiese Journal of New Drugs, 2016,25(05):499-502.

[7]. Minister of Health of the People's Republic of China. Good Manufacturing Practice for Pharmaceutical Products (Amended in 2010). 2011-02-12.

[8]. FDA. Determining the Need for and Content of Environmental Assessments for Gene Therapies, Vectored Vaccines, and Related Recombinant Viral or Microbial Products [S]. 2015-03

[9]. Cassemiro KM, Fernanda MB, Mikaela RF, et al. Molecular and Phenotypic Characterization of a Highly Evolved Type 2 Vaccine-Derived Poliovirus Isolated from Seawater in Brazil, 2014 [J]. Plos One, 11(3):e0152251. 
[10]. Kun Z, Jaune J, Jing S, et al. Are Circulating Type 2 Vaccine-derived Polioviruses (VDPVs) Genetically Distinguishable from Immunodeficiency-associated VDPVs? [J]. Computational and structural biotechnology journal, 2017, 15:456-462.

[11]. Xin YL, Li LJ, Kai XN, et al. Evaluation of environment safety of a Japanese encephalitis live attenuated vaccine $[\mathrm{J}]$. Biologicals : journal of the International Association of Biological Standardization, 2019, 60:36-41.

[12]. Liu X, Zhao X, Na R, et al. The structure differences of Japanese encephalitis virus SA14 and SA14-14-2 E proteins elucidate the virulence attenuation mechanism $[\mathrm{J}]$. Protein \& cell, 2019, 10(2): 149-153.

[13]. Ke YM, Cheng YW, Zhong HW. on the Safety Precautions of High-level Biosafety Laboratories [J]. Disaster Medicineand Rescue (ElectronicEdition), 2017,6(02):112-115.

[14]. Dong GM, Ai PZ, Xiao M, et al. Discussion on Bio-safety for Vaccine Production and GMP Certification Inspection [J]. Chinese Pharmaceutical Affairs, 2011,25(12):11711173.

[15]. Sette A, Rappuoli R. Reverse Vaccinology: Developing Vaccines in the Era of Genomics [J]. Immunity, 2010, 33(4): 530-541.

[16]. Giefing C, Meinke A L, Hanner M, et al. Discovery of a novel class of highly conserved vaccine antigens using genomic scale antigenic fingerprinting of pneumococcus with human antibodies [J]. The Journal of Experimental Medicine, 2008, 205(1): 117-131.
[17]. Coffman R L, Sher A, Seder R A. Vaccine adjuvants: putting innate immunity to work [J]. Immunity, 2010, 33(4): 492-503.

[18]. Liu MA. Immunologic basis of vaccine vectors [J]. Immunity, 2010, 33(4):504-515.

[19]. Germain RN. Vaccines and the future of human immunology [J]. Immunity, 2010, 33(4):441-450.

[20]. FDA. Guidance for Industry for the Evaluation of Combination Vaccines for Preventable Diseases: Production, Testing and Clinical Studies [S]. 1997-04

[21]. Ma H, Galvin T A, Glasner D R, et al. Identification of a novel rhabdovirus in Spodoptera frugiperda cell lines $[\mathrm{J}]$. Journal of virology, 2014, 88(12): 6576-6585.

[22]. Geisler C, Jarvis D L. Rhabdovirus-like endogenous viral elements in the genome of Spodoptera frugiperda insect cells are actively transcribed: implications for adventitious virus detection [J]. Biologicals, 2016, 44(4): 219-225.

[23]. CEBTER FOR DRUG EVALUATION, NMPA. Notice on public consultation of the Technical Guideline on the Investigation of Post-approval CMC Changes to Biological Products (Draft for Comment). [EB/OL]. [2019-11-08].

http://www.cde.org.cn/news.do?method=view $\underline{\text { InfoCommon\&id }=314973}$

[24]. CEBTER FOR DRUG EVALUATION, NMPA. Notice on public consultation of the Technical Guideline on the Investigation of Post-approval Manufacturing Process Changes to Vaccines (Draft for Comment). [EB/OL]. 
[2019-09-02].

http://www.cde.org.cn/news.do?method=view $\underline{\text { InfoCommon\&id }=314926}$

[25]. Xin Z, Min L, Jian HL. Discussion on the DNA Residue Control of Human Rabies Vaccine (vero cell) $[\mathrm{J}]$. Chinese Journal of Biologicals, 2019,32(10):1164-1168.

[26]. Su J, Xin Z, Min L. Analysis on the Packaging Form of Lyophilized Vaccine Diluent and the
Sterility Guarantee Measures [J].Chinese Journal of Biologicals, 2019,32(03):365-368.

[27]. Fang L, Guo ZF. The Status of Drug Manufacturing's Deviation and the Solution [J]. Electromechanical Information, 2011(17): 26-29.

[28]. FDA. Guidance for Industry Investigating Out-of-Specification (OOS) Test Results for Pharmaceutical Production [S]. 2006-10 